\title{
Endoscopic ultrasound (EUS) in pancreatic masses with inconclusive radiologic workup - a case series and short review of the literature
}

\author{
Marcel Razpotnik, Simona Bota, Gerolf Essler, Jutta Weber-Eibel, Markus Peck-Radosavljevic
}

Department of Internal Medicine and Gastroenterology (IMuG), Hepatology, Endocrinology, Rheumatology, Nephrology and Emergency Medicine (ZAE) with Centralized Endoscopy Service, Klinikum Klagenfurt am Wörthersee, Klagenfurt, Austria

\begin{abstract}
Endoscopic ultrasound (EUS) is a very sensitive examination to detect pancreatic masses and can provide useful information in cases where conventional radiologic workup remains inconclusive. We present three cases in which EUS was decisive in establishing the correct diagnosis. Case 1: A 74-year-old female was hospitalized because of acute pancreatitis. Medical history, CT and MRI gave no clue to etiology, but EUS diagnosed a small pancreatic tumor. Case 2: A female patient was admitted because of abdominal pain and weight loss. While MRI suspected a pancreatic tumor, EUS showed typical features of autoimmune pancreatitis. Case 3: A 50-year-old patient was hospitalized with cachexia, ascites and pulmonary embolism. At first, a pancreatic tumor was suspected, but EUS showed a cystic lesion with a solid component (pancreatic pseudocyst).
\end{abstract}

Keywords: endoscopic ultrasound; pancreas tumor; pancreatitis; pancreatic cyst

\section{Introduction}

Endoscopic ultrasound (EUS) is known as a highly sensitive diagnostic technique for pancreaticobiliary and gastrointestinal disorders [1]. In some clinical situations it could be more accurate in establishing the correct diagnosis than computer tomography (CT) or magnet resonance imaging (MRI), especially for the detection of microlithiasis and small pancreatic tumors.

Our aim was to present how EUS was decisive in establishing the correct diagnosis in a series of patients.

Received 13.01.2020 Accepted 12.03.2020

Med Ultrason

2021, Vol. 23, No 1, 107-110

Corresponding author: Prim. Univ. Prof. Dr. Markus Peck-Radosavljevic Department of Internal Medicine and Gastroenterology (IMuG), Hepatology, Endocrinology, Rheumatology, Nephrology and Emergency Medicine (ZAE), Klinikum Klagenfurt am Wörthersee, Klagenfurt, 11 Feschnigstrasse, 9020 Klagenfurt am Wörthersee, Austria Phone: +4346353831103

Fax: +4346353831109

Email:markus@peck.at

\section{Cases report}

Case 1. A 74-year-old female presented with vomiting and mild epigastric pain. The patient denied alcohol or nicotine use. Except for mildly increased total bilirubin $(1.45 \mathrm{mg} / \mathrm{dL})$ and a clearly elevated lipase (1436 $\mathrm{U} / \mathrm{L})$, all other parameters were within the normal range. Contrast-enhanced CT of the abdomen showed only peripancreatic fluid collections (fig 1a). MRI described focal pancreatitis (fig 1b). EUS ruled out the biliary etiology, but a well-defined $1.8 \times 1.2 \mathrm{~cm}$ hypoechoic mass in the pancreas head (fig 1c) was identified. EUS-FNA was performed and histological examination revealed atypical biliary epithelial cells with severe dysplasia. Surgery was performed with histology showing pancreatic adenocarcinoma.

Case 2. A 56-year old female presented with postprandial nausea and weight loss. On clinical examination she experienced a moderate epigastric discomfort. She denied any history of alcohol consumption. Her medication was limited to thyroid hormone replacement therapy due to Hashimoto hypothyroidism. Laboratory 


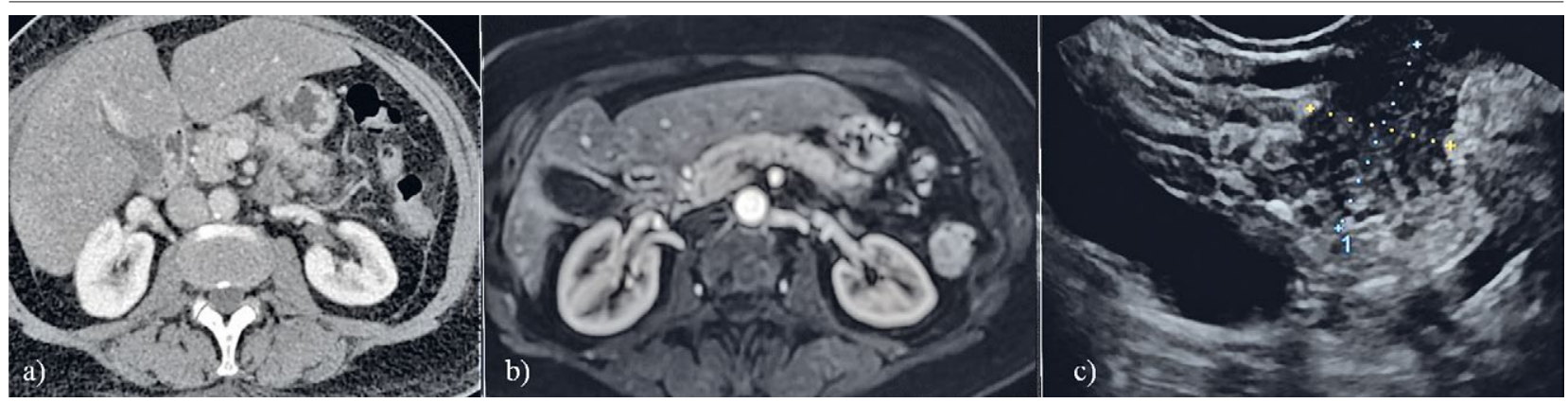

Fig 1. a) normal pancreas on CT; b) focal pancreatitis described on MRI; c) pancreatic tumor detected by EUS.

evaluation showed mild leukocytosis, lipase of $127 \mathrm{U} / \mathrm{L}$ and slightly elevated liver enzymes. On MRI, a pancreatic mass was suspected (fig 2a). Further evaluation with EUS revealed a hypoechoic, $1.7 \mathrm{~cm}$ mass in the pancreas corpus, focal gland enlargement and echogenic interlobar septa, features typical for AIP (fig $2 b$ ). Fibrotic changes with lymphocyte infiltration were present in the histology obtained through EUS-FNA. Even though serum IgG4 was not elevated, an AIP was suspected and steroid therapy started. After 3 months, no pathologic evidence on MRI was found (fig 2c).

Case 3. A 50-year-old male patient with a history of alcohol abuse was hospitalized because of deep vein thrombosis and pulmonary embolism. On physical examination he had cachexia and moderate ascites. The initial blood tests showed normochromic anemia, normal lipase and bilirubin values, lightly elevated GOT, GGT and CRP. CT scan raised the suspicion of a pancreatic mass and liver metastasis (fig 3a), but the following MRI showed no evidence of a solid mass, rather a pancreatic cyst was described. No signs of portal hypertension or liver metastasis were observed and liver cirrhosis was rule out by transient elastography. EUS was performed and a $2.2 \times 1.8 \mathrm{~cm}$ large cystic lesion in the body of the pancreas with solid structures (fig $3 b$ ) was found. FNA was performed and the fluid analysis was typical for a pancreatic pseudocyst (lipase $>120000 \mathrm{U} / \mathrm{L}, \mathrm{CEA}=26.4$
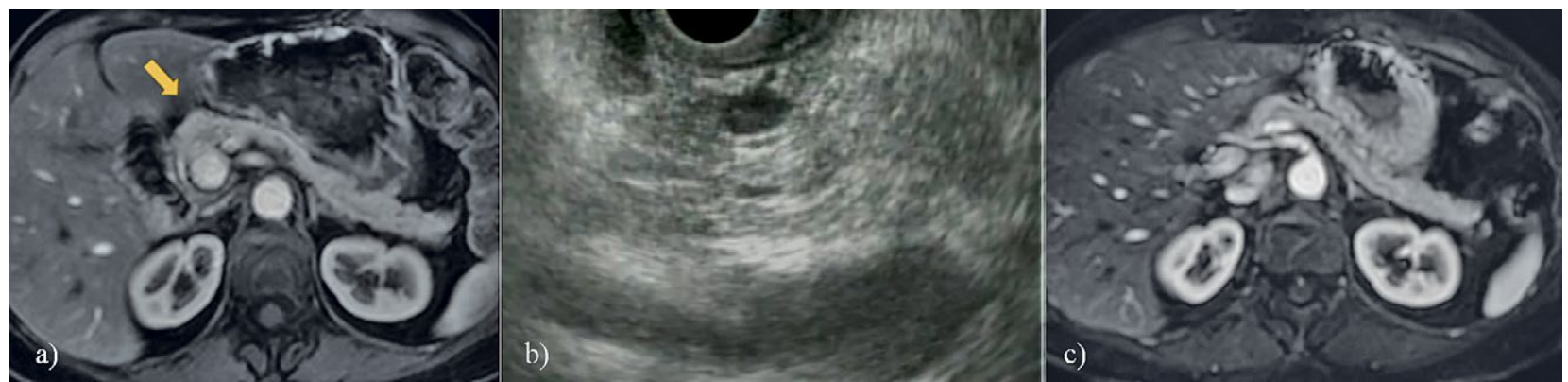

Fig 2. a) MRI suspected a mass in the pancreas neck (arrow); b) EUS shows a $1.7 \mathrm{~cm}$ large mass in the corpus of pancreas, focal gland enlargement and echogenic interlobar septa, features typical for autoimmune pancreatitis; c) normal pancreas on MRI after steroid therapy.

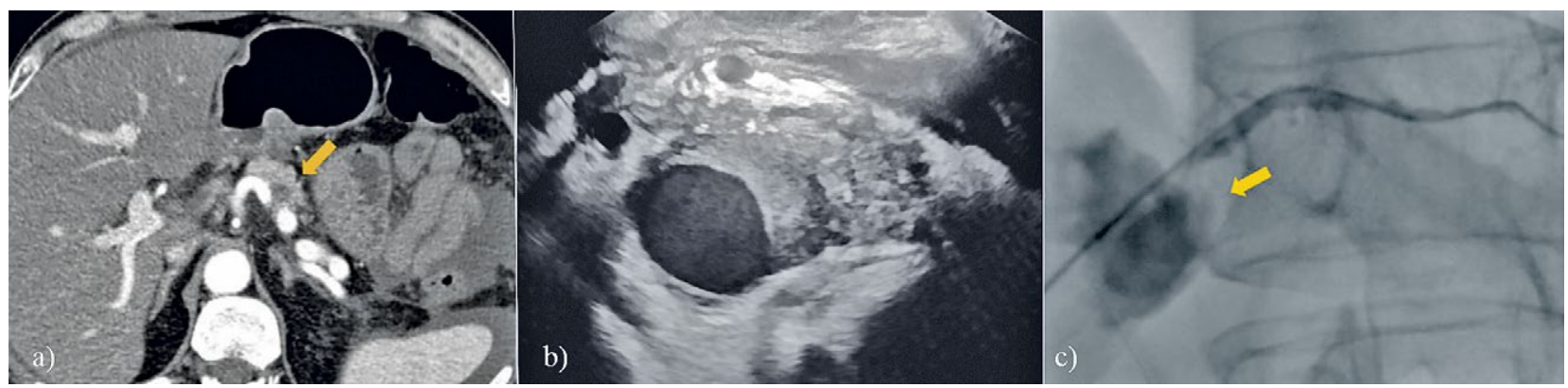

Fig 3. a) CT scan with suspicion of pancreatic tumor (arrow); b) pancreatic cyst with some solid content in EUS; c) pancreatic fistula in ERCP (arrow). 
$\mu \mathrm{g} / \mathrm{L})$. Because of rapid re-occurrence of ascites, a pancreatic duct fistula was suspected and in repeated paracentesis a high level of lipase was observed. Finally, the diagnosis was confirmed by ERCP (fig 3c), during which a plastic stent was placed that was able to control ascites formation right away.

\section{Discussion}

To provide early treatment and prevent further episodes, it is essential to determine the etiology of pancreatitis. In up to $22 \%$ of cases [2] the cause cannot be identified during initial evaluation and recurrences may lead to chronic pancreatitis with limited therapeutic options [3].

A series of studies showed an excellent performance of EUS in the diagnosis of common bile duct stones [1]. Because of the high frequency of the EUS probes and the short distance to the region of interest, high-resolution images can be obtained, which allows better detection of microlithiasis as compared with other diagnostic modalities.

In Case 1 biliary pancreatitis was suspected; $\mathrm{CT}$ and MRI morphological findings were suggestive for pancreatitis but the underlying pathophysiology remained unclear. Diagnostic yield of EUS in cases of idiopathic pancreatitis ranged between 40 and $80 \%[1,4]$. The most frequent etiology were gallstones $(20-60 \%)$, followed by chronic pancreatitis $(2-45 \%)$, pancreatic neoplasia (2$9 \%)$ and ductal anomaly (1-7\%) [4]. The small pancreatic tumor in our patient was not detected on MRI but could be clearly identified on EUS and performance of EUS-FNA secured a definitive diagnosis of malignancy. Current guidelines recommend EUS as the next diagnostic step if routine workup fails to identify the etiology of pancreatitis [5].

Because of the ability to provide high-resolution images, EUS has been considered as more precise than CT and MRI for the diagnosis of small pancreatic lesions [6]. However, conventional EUS shows low specificity (53\%) [1] because most lesions present as hypoechoic and inhomogeneous. Further evaluation with contrastenhanced EUS (CEUS) and elastography is useful to differentiate between benign and malignant masses [7].

AIP can present as a hypoechoic mass mimicking ductal adenocarcinoma as seen in case 2 [8]. Typical appearance of AIP after administration of contrast agent include moderate to marked enhancement of the signal, usually followed by slow washout, while in the case of carcinoma, avascular areas with hypo-enhancement are expected [9]. AIP is a form of chronic fibro-inflammatory disease of the pancreas, which includes two entities, type 1 (multiorgan, IgG4-related) and type 2 (pancreas specific, not IgG4- related) [10]. The inflammatory infiltration periductal can cause narrowing of the distal bile duct and main pancreatic duct, with a typical clinical presentation of painless obstructive jaundice in more than half of the patients. While type 2 AIP affects mainly young patients without a gender predilection, type 1 is more prevalent among older males with possibly systemic IgG4- related autoimmune effects [11].

A definitive diagnosis of AIP can be challenging and is based on a combination of clinical presentation (organ involvement), imaging features, histology, elevated serum IgG4 levels and response to steroids [12]. Typical EUS findings, which suggest an autoimmune process are focal or diffuse "sausage-shaped" enlargement of the pancreas, homogeneous echo pattern, stranding and calcifications. Irregular pancreatic duct with multiple strictures and without associated dilatation can also be present [13].

It is crucial to differentiate AIP from adenocarcinoma, but sometimes it can be challenging [14]. It is very important to evaluate all patients with AIP carefully to rule out an underlying neoplasm. AIP is highly responsive to steroid therapy [15] and in the case of non-response, careful reconsideration of a potential underlying malignancy is recommended.

In general, MRI is the preferred method for the first evaluation of pancreatic cysts. CT scan can be useful when calcifications are present, to differentiate pseudocysts associated with chronic pancreatitis from other etiologies, and to assess vascular involvement and metastases in advanced disease stages [16]. None of the imaging methods is perfect for differentiating between pancreas cysts. EUS is not the first-line examination in these patients but can reveal some additional information and can have a significant impact on the therapeutic concept [17]. CEUS can be performed for assessing the vascularization of the solid components (mural nodule, septa) high vascularization raising the suspicion of malignant transformation within the cyst. In cases of uncertainty and if surgery is planned, FNA should be performed. Cytological analysis of cyst fluid had a low sensitivity $(42 \%)$ and high specificity (99\%) and should be used only in combination with other tests. CEA helps to determine the presence of mucinous cysts but has poor accuracy in distinguishing between benign and malignant cysts [18]. High level of lipase is typically found in pseudocysts. Case 3 presents the importance of the multimodal approach in order to provide optimal care for the patients.

In conclusion, EUS is a useful technique in the clinical practice to establish a correct diagnosis in cases where radiology is inconclusive. 


\section{References}

1. Hawes R. Fockens P. Varadarajulu S. Endosonography 4th Edition. Elsevier, 2018.

2. Zilio MB, Eyff TF, Azeredo-Da-Silva ALF, Bersch VP, Osvaldt $\mathrm{AB}$. A systematic review and meta-analysis of the aetiology of acute pancreatitis. HPB (Oxford) 2019;21:259-267.

3. Somani P, Navaneethan U. Role of ERCP in Patients With Idiopathic Recurrent Acute Pancreatitis. Curr Treat Options Gastroenterol 2016;14:327-339.

4. Jenssen C, vom Lampe B, Kahl S. Endoscopic Ultrasound in Acute Pancreatitis. Video Journal and Encyclopedia of GI Endoscopy 2013;1:554-559.

5. Working Group IAP/APA Acute Pancreatitis Guidelines. IAP/APA evidence-based guidelines for the management of acute Pancreatitis. Pancreatology 2013;13:e1-e15.

6. Volmar KE, Vollmer RT, Jowell PS, Nelson RC, Xie HB. Pancreatic FNA in 1000 cases: a comparison of imaging modalities. Gastrointest Endosc 2005;61:854-861.

7. Iglesias-Garcia J, Lindkvist B, Lariño-Noia J, AbdulkaderNallib I, Dominguez-Muñoz JE. Differential diagnosis of solid pancreatic masses: contrast-enhanced harmonic (CEH-EUS), quantitative-elastography (QE-EUS), or both? United European Gastroenterol J 2017;5:236-246.

8. Notohara K, Burgart LJ, Yadav D, Chari S, Smyrk TC. Idiopathic chronic pancreatitis with periductal lymphoplasmacytic infiltration: clinicopathologic features of 35 cases. Am J Surg Pathol 2003;27:1119-1127.

9. D’Onofrio M, Gallotti A, Principe F, Mucelli RP. Contrast-enhanced ultrasound of the pancreas. World J Radiol 2010;2:97-102.
10. Kloppel G, Detlefsen S, Chari ST, Longnecker DS, Zamboni G. Autoimmune pancreatitis: the clinicopathological characteristics of the subtype with granulocytic epithelial lesions. J Gastroenterol 2010;45:787-793.

11. Kamisawa T, Chari ST, Lerch MM, Kim MH, Gress TM, Shimosegawa T. Recent advances in autoimmune pancreatitis: type 1 and type 2. Gut 2013;62:1373-1380.

12. Chari ST, Smyrk TC, Levy MJ, et al. Diagnosis of autoimmune pancreatitis: the Mayo Clinic experience. Clin Gastroenterol Hepatol 2006;4:1010-1016.

13. Shakov R, DePasquale JR, Elfarra H, Spira RS. Autoimmune Pancreatitis: Case Series and Review of the Literature. Ann Clin Lab Sci 2009;39:167-175.

14. Witkiewicz AK, Kennedy EP, Kennyon L, Yeo CJ, Hruban RH. Synchronous autoimmune pancreatitis and infiltrating pancreatic ductal adenocarcinoma: case report and review of the literature. Hum Pathol 2008;39:15481551.

15. Sah RP, Chari ST, Pannala R, et al. Differences in clinical profile and relapse rate of type 1 versus type 2 autoimmune pancreatitis. Gastroenterology 2010;139:140-148.

16. European Study Group on Cystic Tumours of the Pancreas. European evidence-based guidelines on pancreatic cystic neoplasms. Gut 2018;67:789-804.

17. TanakaM, Fernández-Del Castillo C, Kamisawa T, et al. Revisions of international consensus Fukuoka guidelines for the management of IPMN of the pancreas. Pancreatology 2017;17:738-753.

18. Ngamruengphong S, Bartel MJ, Raimondo M. Cyst carcinoembryonic antigen in differentiating pancreatic cysts: a meta-analysis. Dig Liver Dis 2013;45:920-926. 